\title{
FIRST BISTATIC SPACEBORNE SAR EXPERIMENTS WITH TANDEM-X
}

\author{
Marc Rodriguez-Cassola, Pau Prats, Daniel Schulze, Nuria Tous-Ramon, Ulrich Steinbrecher, \\ Luca Marotti, Matteo Nannini, Marwan Younis, Paco López-Dekker, Manfred Zink, Andreas Reigber, \\ Gerhard Krieger, and Alberto Moreira
}

Microwaves and Radar Institute, German Aerospace Center (DLR), Oberpfaffenhofen, Germany

\begin{abstract}
TanDEM-X is a high-resolution interferometric mission with the main goal of providing a global and unprecedentedly accurate digital elevation model (DEM) of the Earth surface by means of single-pass X-band SAR interferometry. Despite its usual quasi-monostatic configuration, TanDEM-X is the first genuinely bistatic SAR system in space. During its monostatic commissioning phase, the system has been mainly operated in pursuit monostatic mode. However, some pioneering bistatic SAR experiments with both satellites commanded in non-nominal modes have been conducted with the main purpose of testing the performance of both space and ground segments in very demanding scenarios. In particular, this paper reports about the first bistatic acquisition and the first single-pass interferometric (mono/bistatic) acquisition with TanDEM-X. Even in the absence of essential synchronisation and calibration information, bistatic images and interferograms with similar quality to pursuit monostatic have been obtained.
\end{abstract}

Index Terms - TanDEM-X, spaceborne SAR missions, time and phase synchronisation

\section{INTRODUCTION}

TanDEM-X (TDX) is the first bistatic SAR system in space, whose main goal is the generation of a global digital elevation model (DEM) following the HRTI-3 standard [1]. TanDEM$\mathrm{X}$ extends the TerraSAR-X (TSX) mission by adding a second fully-active satellite with cooperative operation capabilities forming a single-pass interferometric system, and is therefore capable of providing very accurate $3 \mathrm{D}$ information. During the first years of the TerraSAR-X mission, DLR has benefitted from the flexibility of the system to perform some innovative SAR experiments in non-nominal configurations, i.e., spaceborne-airborne bistatic imaging [2] or the demonstration of new imaging modes [3]. The uniqueness and the increased operational possibilities of the new mission allow one to envisage a large amount of challenging and creative experiments to take advantage of the potential of the payloads. Besides the

The TanDEM-X project is partly funded by the German Federal Ministry for Economics and Technology (Förderkennzeichen $50 \mathrm{EE} \mathrm{1035).}$ obvious goal of testing the performance of the system in nonnominal bistatic configurations, a further objective should be outlined: develop and test new techniques, modes or algorithms which might become relevant in future SAR missions.

The paper addresses the first bistatic experiments performed with TanDEM-X during its monostatic commissioning phase: 1) the first bistatic acquisition, complemented with a repeat-pass interferometric processing of consecutive bistatic surveys, and 2) the first single-pass bistatic interferometric acquisition. The paper is structured as follows. Section 2 describes the aspects of the monostatic commissioning phase relevant to the conduction of these innovative experiments. Section 3 presents a detailed description of the experiments and results. Section 4 concludes the paper with a summary.

\section{TANDEM-X IN THE PURSUIT MONOSTATIC COMMISSIONING PHASE}

The TDX satellite was launched from Baikonur cosmodrome on June 21st, 2010. For approximately one month, TDX followed a specific test schedule while approaching TSX from the original $16000 \mathrm{~km}$ to the final flight formation with 20 $\mathrm{km}$ separation planned for the monostatic commissioning phase. For the following months, both satellites operated independently in pursuit monostatic mode, TDX completing a monostatic test program to validate its expected performance. The monostatic commissioning phase ended in early October 2010, after which TDX approached TSX to a distance of some hundred meters to achieve the so-called close flight formation.

Despite the tight operational schedule, some room was reserved for innovative experiments during the first months of the mission. The monostatic commissioning phase was very appealing to perform non-nominal bistatic SAR experiments because of the longer along-track baselines. It has been under these circumstances that the first bistatic experiments with TanDEM-X have been conducted. Fig. 1 depicts the reference configuration formed by the TSX and TDX satellites at the period. 


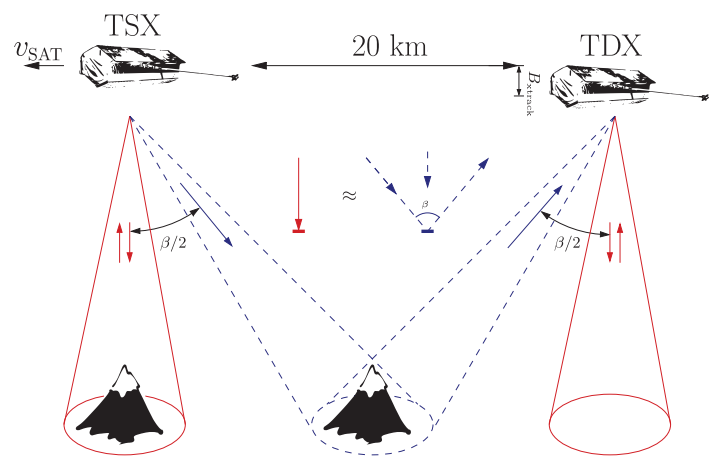

Fig. 1. Formation of the TSX and TDX satellites during the TanDEM-X monostatic commissioning phase.

Table 1. Acquisition parameters

\begin{tabular}{l|cc}
\multicolumn{2}{c}{ Table 1. Acquisition parameters } & \\
& Experiment 1 & Experiment 2 \\
\hline PRF [Hz] & 3182.52 & $2991.24 \times 2$ \\
Tx bandwidth $[\mathrm{MHz}]$ & 100 & 150 \\
Incident angle $[\mathrm{deg}]$ & 36.6 & 35.8 \\
Squint angle TSX/TDX $[\mathrm{deg}]$ & \pm 0.8 & \pm 0.9 \\
Cross-track baseline $[\mathrm{m}]\left(B_{\text {xtrack }}\right)$ & 253 & 43 \\
\hline
\end{tabular}

\section{EXPERIMENTAL RESULTS}

As previously stated, two sets of innovative bistatic experiments have been carried out during the monostatic commissioning phase. In both cases, the geometrical configuration coincided with the one depicted in Fig. 1: the beams used in pursuit monostatic operation are represented by solid lines, corresponding the dashed ones to a bistatic operation with symmetric azimuth steering. Table 1 lists the main parameters of the acquisitions. The column 'Experiment 1' refers to the first bistatic imaging acquisition, as well as to the repeatpass bistatic interferometric results; the column 'Experiment 2' refers to the first single-pass bistatic interferometric acquisition. All data-takes were acquired using the regular stripmap modes of the satellites. The data have been processed using the experimental TanDEM-X interferometric processor (TAXI), a flexible and versatile processing suite especially developed for the evaluation of TanDEM-X experimental data products [4].

\subsection{Bistatic imaging and repeat-pass interferometry}

The acquisition, carried out on August 8th, 2010, was planned over Brasilia city. For this first bistatic experiment, TSX operated monostatically with a squint of $-0.8 \mathrm{deg}$, whereas TDX was set in receive-only mode with a squint of $0.8 \mathrm{deg}$. Due to the small bistatic angle, no relevant modifications of the timing schemes were required. Synchronisation pulses were exchanged during the data-take, from which the clock phase er-

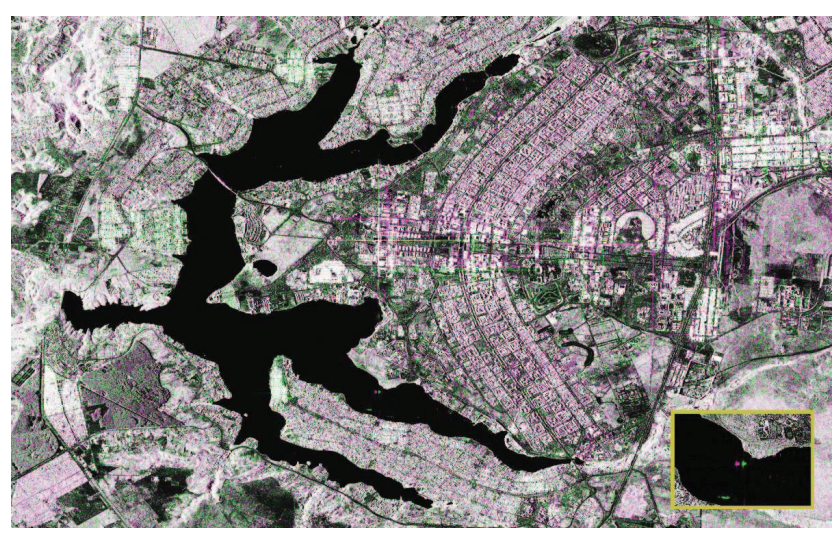

Fig. 2. TDX first bistatic image (green channel) over Brasilia. TSX monostatic image (magenta channel) overlaid. Radar coordinates (horizontal range, vertical azimuth time).

ror could be measured [1]. A squinted monostatic image and a non-squinted bistatic one were obtained, but with no spectral overlap between them. Similar acquisitions were conducted in consecutive passes of the system over the same area to produce bistatic repeat-pass interferograms, i.e., after 11 days.

The first bistatic image acquired by TanDEM-X is shown in Fig. 2 (green channel), overlaid with the simultaneous TSX monostatic image (magenta channel). Two different aspects of the previous images can be outlined. In the city centre, the dominant scattering mechanism seems to be monostatic, but there exist distinct building areas near the lake, where the bistatic scattering dominates. The conclusion is that, even for the small bistatic angle of the experiment, about $1.6 \mathrm{deg}$, significant changes in target reflectivity, especially in man-made structures, can be expected between monostatic and bistatic observations. This feature might be very helpful to enhance the performance of existing identification or classification algorithms. The second one is the distribution of the azimuth ambiguities. Considering the azimuth ambiguities of the point target of opportunity in the centre of Fig. 2, which are mapped on the lake and zoomed within the ochre rectangle on the bottom right corner of the figure, a range difference in the position of the monostatic and bistatic ambiguities appear. This happens because the monostatic image is squinted, whereas the bistatic is not, and therefore the 2D monostatic impulse response, unlike the monostatic, is skewed. This feature might be exploited to develop ambiguity identification/supression strategies. Fig. 3 shows the repeat-pass interferogram using the two bistatic images after eleven days. Note that the images are rotated $90 \mathrm{deg}$ with respect to Fig. 2. The figure on the left shows the non-synchronised bistatic interferogram. The azimuthal fringes of the interferogram are due to the differential clock frequency change between the two passes, of about $1 \mathrm{~Hz}$. The right figure shows the synchronised interferogram; note that the SRTM DEM has been used in Fig. 3 to 


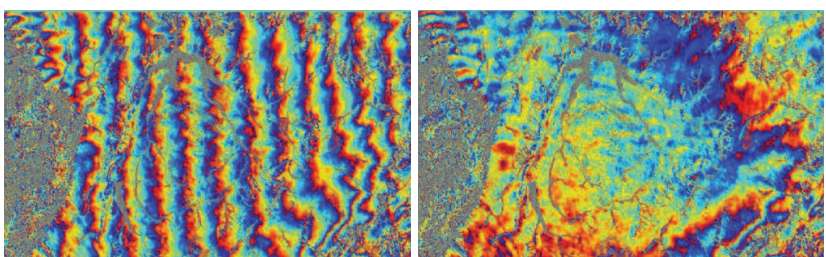

Fig. 3. Results of bistatic 11-day repeat-pass interferometry with TanDEM-X.

remove topography. The residual fringes correspond mainly to a priori DEM errors and (possibly) marginally to unaccounted atmospheric effects. The mean value of the coherence is 0.35 ; in urban areas, this value increases to about 0.5 . There are no significant differences between the values obtained from the monostatic repeat-pass and the bistatic interferograms. Concerning the interferometric performance, the baselines are practically the same, as is the SNR of both acquisitions. Note that the monostatic image has a squint, but since no significant changes in target reflectivity other than for certain man-made structures have been observed, the results are definitely consistent. Besides its novelty, the relevant conclusion of this experiment was that we could obtain with the new system bistatic images and interferograms of similar quality to the monostatic (more mature) TerraSAR-X counterparts, a quite relevant information at the time.

\subsection{Bistatic single-pass interferometry}

Following the success of the bistatic imaging acquisitions, a natural next experiment consisted of performing a single-pass bistatic interferometric experiment with a large along-track baseline before the end of the pursuit monostatic commissioning phase. However, a way to overcome the spectral decorrelation of the previous bistatic configuration was needed. Because of the small bistatic angle, simultaneous monostatic and bistatic images with similar equivalent squint angles have Doppler spectral overlap, i.e., the images are coherent. This equivalency is depicted in Fig. 1. To achieve this, an imaginative commanding of the satellites was designed, with a switch of the azimuth antenna patterns of TSX and TDX on a pulseto-pulse basis. Both satellites transmitted one pulse using the non-squinted beams (solid lines) in Fig. 1; in the next pulse TSX transmitted with a squint of -0.9 deg and TDX only received with a squint of +0.9 deg (depicted with dashed lines in Fig. 1). All things considered, one pursuit monostatic interferogram with full baseline, plus two symmetric bistatic interferograms with half baseline could be computed. However, the acquisition had a couple of drawbacks: firstly, the PRF needed to be doubled, i.e., the swath was halved; secondly, due to the specifics of the commanding, no calibration nor synchronisation pulses were available. The acquisition

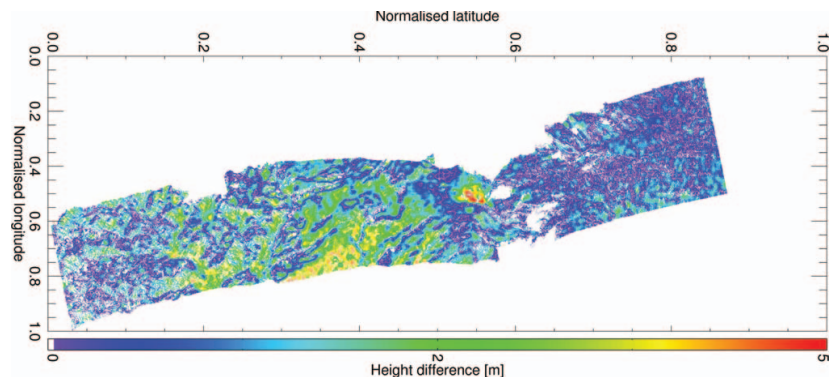

Fig. 5. Difference between pursuit monostatic and single-pass bistatic DEMs. Effective averaging factor is roughly 20.

was carried out over the Parque nacional del volcán Turri$a l b a$, in Costa Rica, a gracefully montainous area. Note that this experiment was conducted early October 2010, about a week before the first official bistatic TanDEM-X interferograms in close formation were obtained, and are therefore the first bistatic single-pass spaceborne SAR interferometric acquisitions.

As stated before, TDX uses a direct X-band link to measure the differential phase between the two radar master clocks. For the cases where this synchronisation information is missing, TAXI has an automatic synchronisation module which is capable of estimating the synchronisation error using the bistatic data $[5,6]$, which definitely substantiates the scientific character of the experiment. The estimated clock carrier frequency difference of about $124 \mathrm{~Hz}$ is also consistent with available contemporary SyncLink samples. Fig. 4 shows the DEM generated using one of the bistatic interferograms. We can show the validity of the approach by cross-checking the results obtained using the single-pass bistatic interferogram of Fig. 4 and the one resulting from the conventional pursuit monostatic one. Fig. 5 shows the height difference between the two DEMs. A mask has been used to avoid including values with low coherence. No trends in range or azimuth can be identified, which qualitatively validates the automatic synchronisation approach. The standard deviation in the height error of the DEMs computed using single-look interferograms corresponds to $23.3 \mathrm{~m}$, which results in an effective averaging factor of about 20 for the DEM error of the figure.

Fig. 6 shows a crop of the pursuit monostatic (left) and one bistatic (right) flattened interferogram showing an area near the volcano. As expected, the pursuit monostatic interferogram has twice as much height sensitivity as the bistatic. The height of ambiguity of the pursuit monostatic acquisition is of about $85.14 \mathrm{~m}$. In terms of coherence, we expect the mono-bistatic pairs to be less sensitive to volume decorrelation, because of the halved baseline. Nevertheless, the bistatic image is also expected to have an SNR $1.2 \mathrm{~dB}$ worse for equivalent reflectivity. This loss in SNR is attributed mainly to the electronic antenna steering and consequently causes 


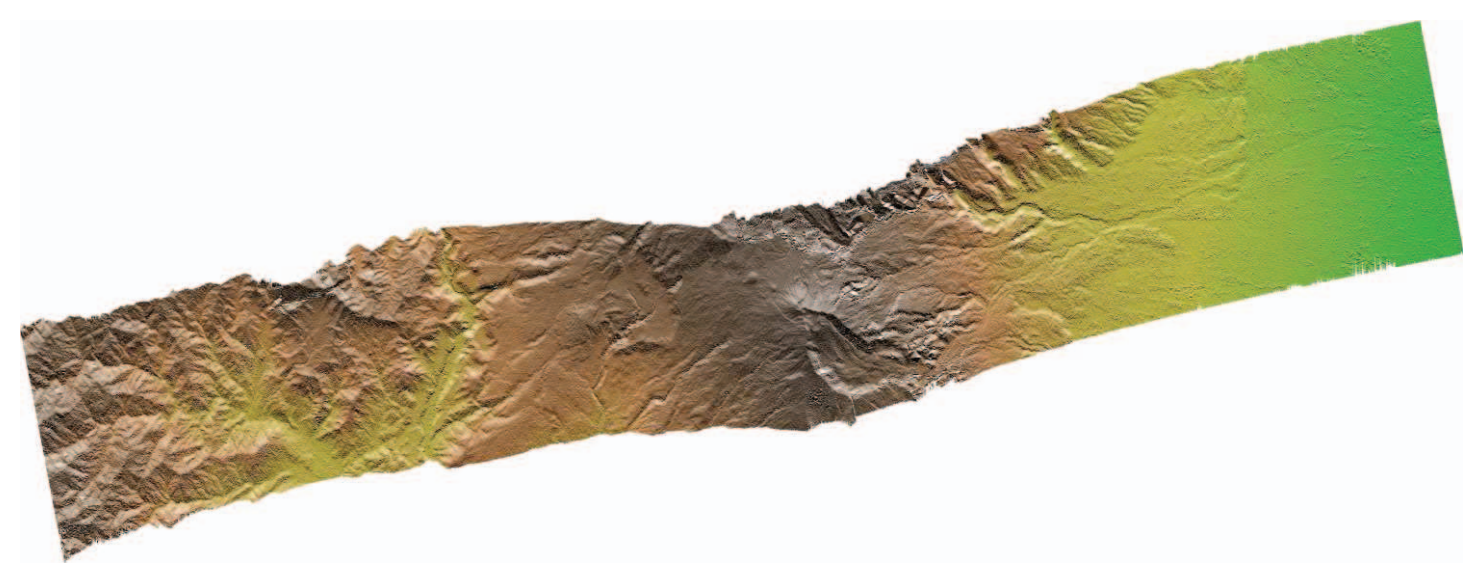

Fig. 4. Geocoded (North points rightwards) DEM of the are sorrounding Turrialba volcano, the first single-pass bistatic interferometric TanDEM-X acquisition.
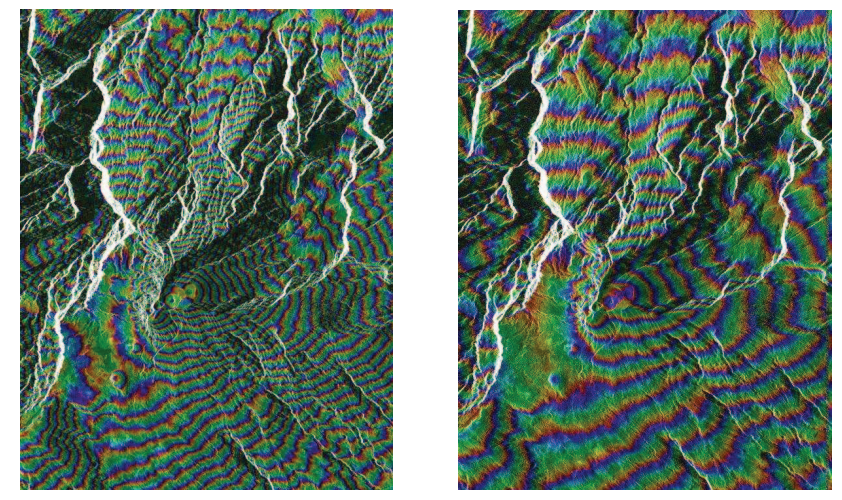

Fig. 6. Crop of the interferograms in the areas sorrounding the volcano. Pursuit monostatic (left) and single-pass bistatic (right) interferograms.

a proportional loss of the overall coherence in the monobistatic pairs. The mean value of the coherence of the pursuit monostatic pair is 0.645 ; for the single-pass bistatic pairs this value drops to about 0.625 . To illustrate the impact of volume decorrelation in the pursuit monostatic and one single-pass bistatic acquisitions, Fig. 7 shows the interferometric coherences (middle pursuit monostatic, right single-pass bistatic) in a forest area north from the volcano; the left crop corresponds to the bistatic intensity image. Though not blatantly evident, the forest area has a lower coherence in the pursuit monostatic pair due to volumetric decorrelation.

\section{SUMMARY}

We have presented two innovative experiments performed with TanDEM-X in the pursuit monostatic commissioning phase of the mission: the first bistatic imaging acquisitions and the first single-pass bistatic interferometric acquisition.

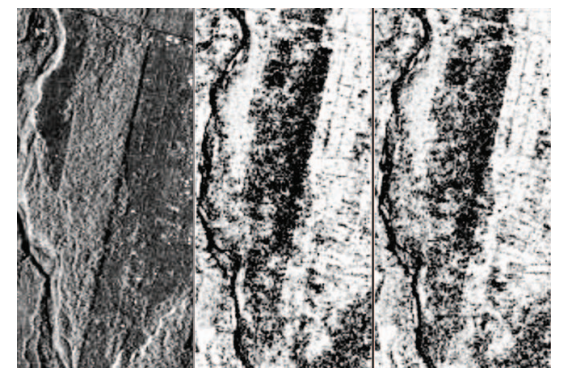

Fig. 7. Forest area north from the volcano to note different volumetric decorrelation. Intensity (left), pursuit monostatic coherence (middle), and bistatic coherence (right).

\section{REFERENCES}

[1] G. Krieger, et al., 'TanDEM-X: a satellite formation for high-resolution SAR interferometry', IEEE Trans. Geosci. Remote Sens., vol. 45, no. 11, Nov. 2007.

[2] M. Rodriguez-Cassola, et al., 'Bistatic TerraSAR-X/F-SAR spaceborne-airborne experiment: description, data processing and results', IEEE Trans. Geosci. Remote Sens., vol. 48, no. 2, Feb. 2010 .

[3] P. Prats, et al., 'Processing of Sliding Spotlight and TOPS SAR Data Using Baseband Azimuth Scaling', IEEE Trans. Geosci. Remote Sens., vol. 48, no. 2, Feb. 2010.

[4] P. Prats, et al., TAXI: A Versatile Processing Chain for Experimental TanDEM-X Product Evaluation, Proc. IGARSS, 2010, Honolulu, USA.

[5] M. Rodriguez-Cassola, et al., A Versatile Processing Chain for Experimental TanDEM-X Product Evaluation, Proc. EUSAR, 2010, Aachen, Germany.

[6] M. Rodriguez-Cassola, et al., General Processing Approach for Bistatic SAR Systems: Description and Performance Analysis, Proc. EUSAR, 2010, Aachen, Germany. 